\title{
Knee Osteoarthritis after Reconstruction of Isolated Anterior Cruciate Ligament Injuries: A Systematic Literature Review
}

\author{
Compagnoni Riccardo ${ }^{1}$ Catani Fabio ${ }^{2}$ Randelli Pietro ${ }^{1,3}$ \\ ${ }^{1}$ ASST Centro Specialistico Ortopedico Traumatologico Gaetano Pini - \\ CTO, Milan, Italy \\ 2 Department of Orthopaedic Surgery, Azienda Ospedaliero- \\ Universitaria Policlinico di Modena, University of Modena and \\ Reggio-Emilia, Modena, Italy \\ 3 Dipartimento di Scienze Biomediche per la Salute, University of \\ Milan, Milan, Italy
}

Joints 2017;5:39-43.

\author{
Address for correspondence Compagnoni Riccardo, MD, ASST Centro \\ Specialistico Ortopedico Traumatologico Gaetano Pini - CTO, Piazza \\ Cardinal Ferrari, 1, 20122 Milan, Italy \\ (e-mail: riccardo.compagnoni@gmail.com).
}

\begin{abstract}
Keywords

- cruciate ligament

- anterior

- osteoarthritis

- knee

- surgical procedures

- operative

Purpose The aim of this review was to analyze the current literature on osteoarthritic evolution of knees without any combined meniscal or ligament lesions undergoing anterior cruciate ligament $(\mathrm{ACL})$ reconstruction.

Methods A PubMed/MEDLINE research was performed using the following keywords: "Anterior Cruciate Ligament Reconstruction" [Mesh] AND "Osteoarthritis, Knee" [Mesh]. Only English language literature and articles published after 2005 were included. Studies including concomitant meniscal tears, posterior cruciate or collateral ligament injuries, previous surgery in the affected knees, infections, osteochondral defects, loose bodies, synovial plica syndrome, and posteromedial or posterolateral corner injuries were not considered in this review.

Results Twelve studies were selected. These papers included 892 patients (mean age at the time of surgery was 22.3 years), with an average follow-up of 11 years. Imaging at follow-up was obtained with standard radiographs in nine studies, magnetic resonance imaging (MRI) in one study, and both X-rays and MRI in two studies. Eight studies reported osteoarthritic evolution, with different prevalence.

Conclusion Only few high-quality studies focused on these specific patients have been published. When reconstructed, isolated ACL-deficient knees have a low risk of osteoarthritic evolution, but mild signs of joint degeneration are reported by the current literature. Level of Evidence Level IV, systematic review of level I to level IV studies.
\end{abstract}

\section{Introduction}

Anterior cruciate ligament (ACL) is one of the most commonly injured ligaments of the knee. Professional athletes in basketball, soccer, and other contact sports report an annual incidence of 0.15 to $3.7 \%$ of ACL injury, with an higher rate in female population. ${ }^{1,2}$ Thirty-five years ago, it was stated that a rupture of the ACL was "the beginning of the end" of the knee. ${ }^{3}$

Controversy still exists concerning the proper treatment of a knee with a deficient ACL. The current literature reports a large number of studies describing different surgical tech- niques and comparing results of these treatments. Many of these papers recommend earlier surgical reconstruction after ACL injury to prevent further meniscal damage and to decrease the risk of degenerative arthritis. ${ }^{4-6}$ During the last years, a small number of studies compared the results of surgical and conservative treatment of acute ACL injuries in young population. ${ }^{7}$ Medial and lateral meniscal tears are described as a risk factor for osteoarthritis $(\mathrm{OA})$ in the injured knee. ${ }^{8}$ Many factors are not assessed or adequately evaluated by several studies, such as age, gender, meniscal tear pattern, mechanical alignment, activity level, combined ligament 
injuries, and previous surgery. Also, a persistent and evolving disturbance in cytokine and keratin sulfate profiles was also observed in ACL-deficient knees compared with uninjured knees. $^{9,10}$ These confounding factors have a clinical relevance, and a systematic review analyzing the risk of knee $\mathrm{OA}$ after ACL reconstruction in isolated ACL injuries has not yet been reported in current literature.

Aim of this review was to analyze the current literature on osteoarthritic evolution of knees without any combined meniscal or ligament lesions undergoing ACL reconstruction.

\section{Methods}

A systematic review of scientific articles listed in medical databases (PubMed, MEDLINE) was performed in February 2016, according to the PRISMA (Preferred Reporting Items for Systematic Review and Meta-Analysis) guidelines. ${ }^{11}$ The search for relevant articles was performed using the following key words: "Anterior Cruciate Ligament Reconstruction"[Mesh] AND “Osteoarthritis, Knee"[Mesh]. English language literature and article published after 2005, involving also groups of patients with previous traumas were considered. Only clinical studies with radiographic results evaluated up to the final follow-up were considered, whereas those without controls over time were excluded. If a case series was included in more than one article, the one with the longest follow-up was considered. When two series of patients were described in the same article, only the one respecting inclusion criteria was analyzed. Original scientific prospective or retrospective articles with a level of evidence of I to IV were included. Review studies, expert opinions, book chapters, and abstracts of meetings or scientific conferences were excluded. Studies including concomitant meniscal tears, posterior cruciate or collateral ligament injuries, previous surgery in the affected knees, infections, osteochondral defects, loose bodies, synovial plica syndrome, and posteromedial or posterolateral corner injuries were not considered in this review. If in a study a cohort of patients respecting inclusion and exclusion criteria was found and compared with another cohort of patients with collateral injury, the study was included but only patients respecting inclusion and exclusion criteria were analyzed.

Clinical data such as mean age, gender, affected limb, dominant limb, mean period between trauma and the intervention, sports activity, type of treatment, complications, clinical scores and radiological evaluation at follow-up were collected.

The primary outcome of this review was to analyze the osteoarthritic evolution of the knee that underwent an ACL reconstruction.

\section{Results}

The initial literature search revealed 119 potentially relevant papers. All exclusion criteria were applied to these selected studies, and 12 studies were selected for inclusion in this review (-Table 1). Four studies focused on isolated ACL reconstruction without any other injury. The remaining papers compared a cohort of patients with isolated ACL tear with patients having a meniscal tear; for those studies, only data from isolated ACL-injured patients were collected.

The studies included 892 patients, with an average followup of 11 years. Men age at the time of surgery was 22.4 years at the time of surgery. Imaging at follow-up was obtained with standard radiographs in nine studies, magnetic resonance imaging (MRU) in one study, and both X-rays and MRI in two studies.

The International Knee Documentation Committee (IKDC) scale was used in three studies, the Kellgren-Lawrence classification of OA was used in four studies, and the JagerWirth classification was used in one study; the other papers only demonstrated generic signs of articular degeneration. When MRI was performed, the International Cartilage Research Society (ICRS) guidelines or the Haughom scale was used. One paper compared results of surgical and conservative treatment.

Kievit et al $^{12}$ demonstrated an osteoarthritic evolution in patients with isolated ACL tears at a mean follow-up of 5.1 years, but this degenerative process was much faster in patients with correlated meniscal injuries. Data on severity of OA were not reported in the study, and a generic indication about cartilage status was described. ${ }^{12}$

Jones et $\mathrm{al}^{13}$ considered a cohort of 159 patients who underwent ACL reconstruction for an isolated ACL tear and compared with 103 patients with associated meniscal tear. At an average follow-up of 2.3 years, they found no signs of OA in isolated ACL tears. The results of this study are similar to those reported by Lidén et $\mathrm{al}^{14}{ }^{14}$ Hoffelner et $\mathrm{al},{ }^{15}$ and Struewer et al. ${ }^{16}$

Kessler et al ${ }^{17}$ compared results of surgical and conservative treatment in isolated ACL tears. No differences were found in osteoarthritic evolution between the two groups at an average follow-up of 11.1 years. Only $45 \%$ of patients that underwent surgical reconstruction had a KellgrenLawrence grade 0 , compared with $61 \%$ of patients treated conservatively. Results of this study evidenced an osteoarthritic evolution without any advantage for the ones who underwent surgical reconstruction. ${ }^{17}$

Aït Si Selmi et al $^{18}$ reported on a cohort of 44 patients at an average follow-up of 17.4 years, evaluated with the IKDC scale. They observed an osteoarthritic evolution in $13 \%$ of isolated ACL-reconstructed knees compared with $37.2 \%$ of patients with associated meniscal injuries.

Leiter et al $^{19}$ analyzed 68 patients with reconstructed ACL using hamstrings tendon at an average follow-up of 14.6 years. The authors concluded that reconstructed knees have a greater incidence and severity of OA than nonreconstructed knees, which suggests that degenerative changes are secondary to ACL rupture. Medial meniscus surgery was a strong predictor of $\mathrm{OA}$.

Zaid et $\mathrm{al}^{20}$ evidenced on MRI scans that altered tibial position in the ACL-reconstructed knee correlates to degenerative cartilage changes in the contact areas of the medial compartment of the knee, as early as one year following ACL reconstruction. This relationship suggests that alteration in tibial position following ACL reconstruction is one of the 


\begin{tabular}{|c|c|c|c|c|c|c|c|c|c|c|c|c|}
\hline 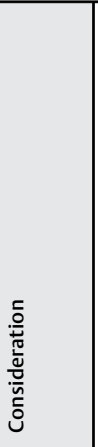 & 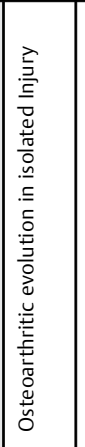 & 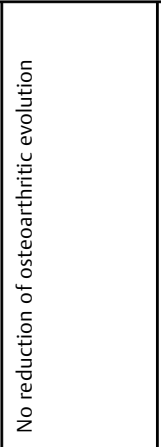 & 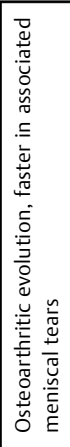 & 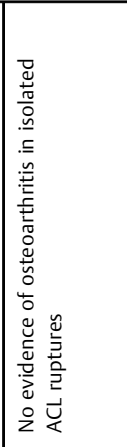 & 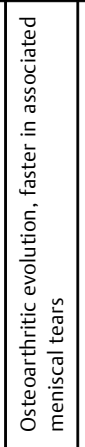 & 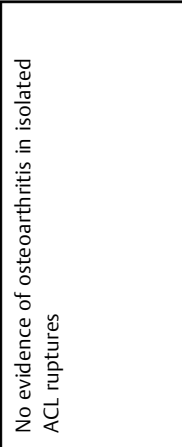 & 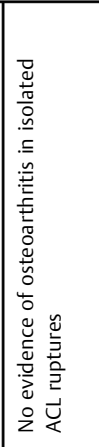 & 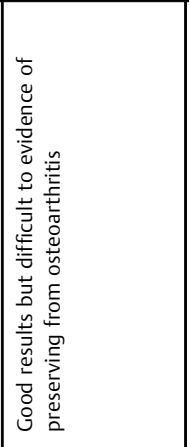 & 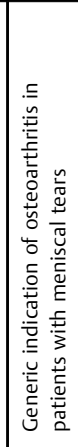 & 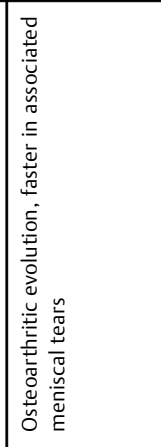 & 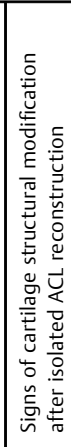 & 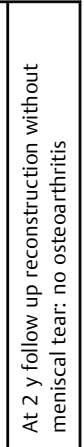 \\
\hline 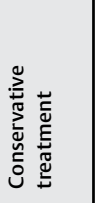 & 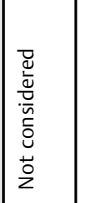 & 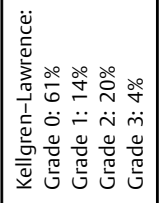 & 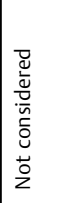 & 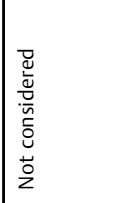 & 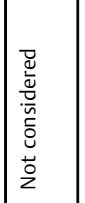 & 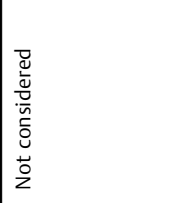 & 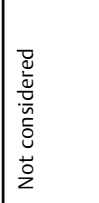 & 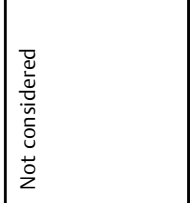 & 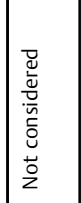 & 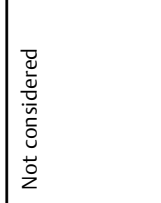 & 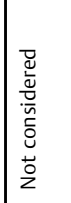 & 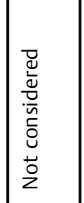 \\
\hline 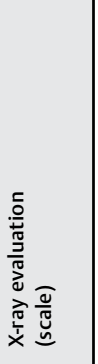 & 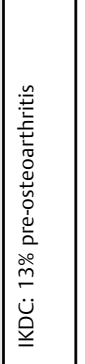 & 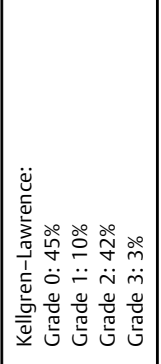 & 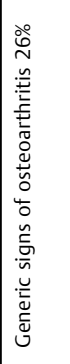 & 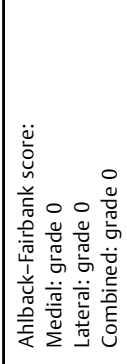 & 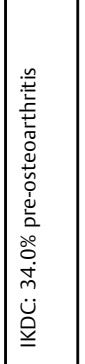 & 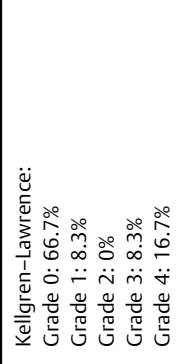 & 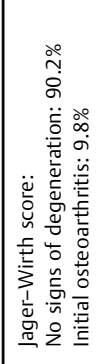 & 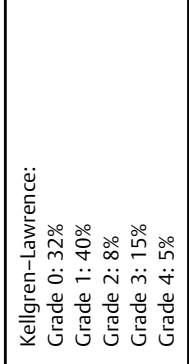 & 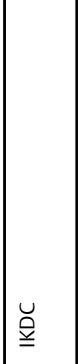 & 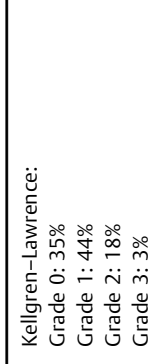 & 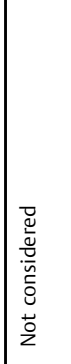 & 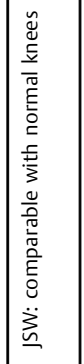 \\
\hline 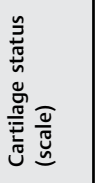 & 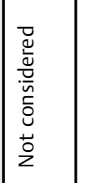 & 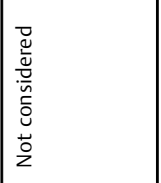 & 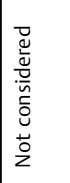 & 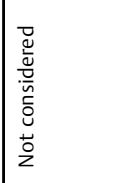 & 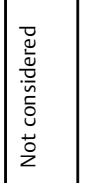 & 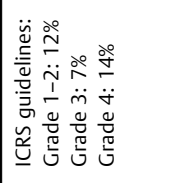 & 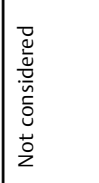 & 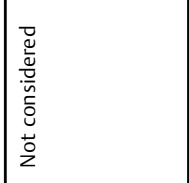 & 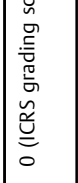 & 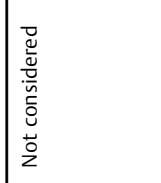 & 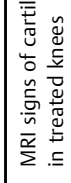 & 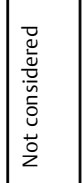 \\
\hline$\sum_{\infty}$ & 2 & \& & q & 2 & 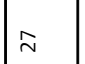 & $\stackrel{\text { N }}{\Delta}$ & z & z & $\stackrel{\sim}{\sim}$ & $\stackrel{\infty}{\sim}$ & $\approx$ & $\bar{\sim}$ \\
\hline 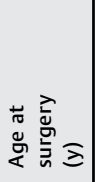 & 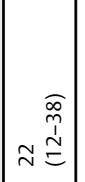 & 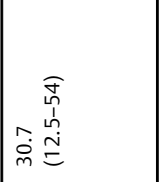 & 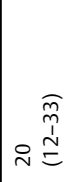 & 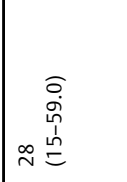 & 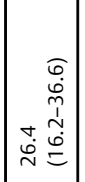 & 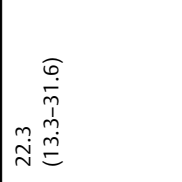 & 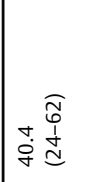 & 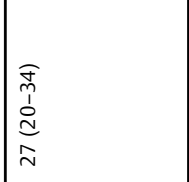 & 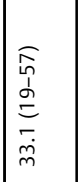 & 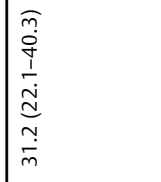 & 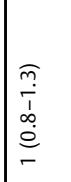 & $\stackrel{\circ}{m}$ \\
\hline 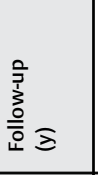 & 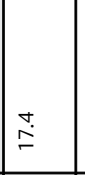 & 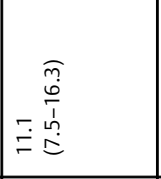 & 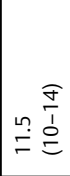 & 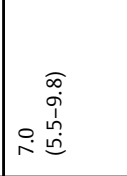 & 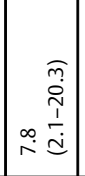 & 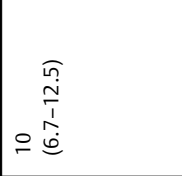 & 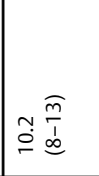 & 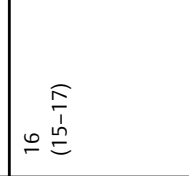 & 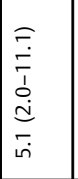 & 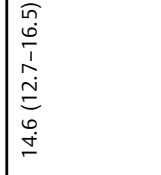 & 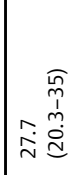 & 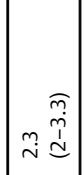 \\
\hline 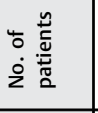 & \& & 8 & g & $\stackrel{m}{m}$ & $\stackrel{\sim}{\sim}$ & $\stackrel{\infty}{\sim}$ & 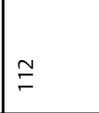 & 0 & $=$ & 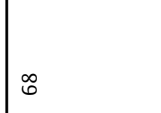 & 足 & 量 \\
\hline 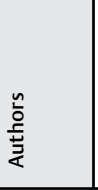 & 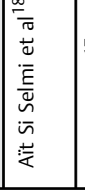 & 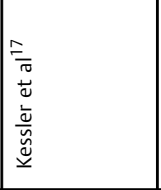 & 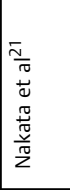 & 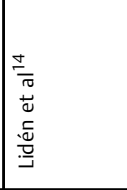 & 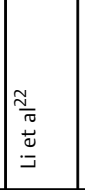 & 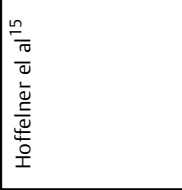 & 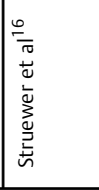 & 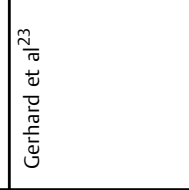 & 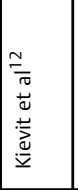 & 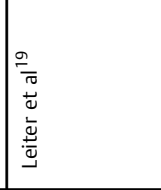 & 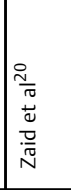 & 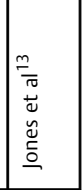 \\
\hline
\end{tabular}


mechanisms for the accelerated cartilage changes commonly seen following ACL reconstruction.

Nakata et ${ }^{21}$ reported on a cohort of 46 patients at an average follow-up of 11.5 years. They found generic signs of OA in 26\% of patients with reconstructed ACL without meniscal tears and $86 \%$ in patients with associated meniscal tears. There was a statistically significant difference in the incidence of radiographic degenerative joint changes between meniscus-preserved knees and meniscus excised knees. Similar results were found in a study performed by Li et $\mathrm{al}^{22}$ on a cohort of 212 patients at an average follow-up of 26.4 years and in a study performed by Gerhard et $\mathrm{al}^{23}$ on a cohort of 63 patients at an average follow-up of 16 years.

\section{Discussion}

ACL reconstruction is a reproducible technique and in the recent years has become the gold standard treatment for injured knees in active, young population. The aim of this surgery is to restore the joint stability and to protect the knee from further meniscal and ligament injuries. One of the prospected advantages of ACL reconstruction is to restore the correct knee biomechanics, preventing also the articular cartilage degeneration that might evolve in knee OA.

As evidenced by many studies in the literature, meniscal tears are a positive predictor of knee osteoarthritic degeneration due to reduced contact area between femoral and tibial cartilage. Meniscal tears, especially medial posterior horn tears, can also reduce articular stability and increase osteoarthritic evolution. ${ }^{24,25}$ Association between meniscal and ACL tears is a common finding in clinical practice, and understanding the influence of the single lesion on evolution of the articular environment is not immediate. The aim of this systematic review was to evidence the up-to-date literature trying to identify the influence of an isolated ACL tear on osteoarthritic evolution of the knee.

Only a small number of studies analyzing the radiographic or MRI results at medium- and long-term follow-up for reconstructed isolated ACL tears are available in the literature of the past 10 years. These studies have different outcomes, showing no evolution to $\mathrm{OA}^{14}$ or signs of cartilage degeneration on MRI scans 10 years after surgery. ${ }^{20}$

Eight papers described the osteoarthritic evolution comparing isolated ACL tears with combined meniscal or ligament lesions. Jones et al reported no degenerative evolution in patients without meniscal tears, compared with a clear osteoarthritic degeneration in patients with meniscal lesions. These results seem to be confirmed by Lidén et al. ${ }^{14}$ Different conclusions are described by Nakata et $\mathrm{al}^{21}$ and Aït Si Selmi et al, ${ }^{18}$ who evidenced a degenerative evolution of the knees in isolated ACL tears, even if not so pronounced as in the knees with meniscal tears.

The use of different scales (IKDC, Joint Space Width, ICRS, Ahlback and Fairbank score, and Kellgren-Lawrence score) makes it difficult to compare exactly the results of different studies and to perform a pooled data analysis. Moreover, the papers do not provide clear information about interobserver reliability of the outcome measurements. In addition, meniscal repairs were included in the noninjured meniscus group in some papers and in the injured meniscus group in others.

Analysis of collected data evidenced a clear indication that meniscal lesions, when combined with ACL rupture, elevate the risk of OA. Isolated ACL tears have a low risk of osteoarthritic evolution, but signs of degeneration are reported in different studies. Only two studies excluded cartilage degeneration in isolated ACL tears, but some limits can be recognized in both studies. Jones et $\mathrm{al}^{13}$ did not use an international scale to describe their results but only the joint space narrowing. The strength of this paper is the big number of patients included in the study. Lidén et $\mathrm{al}^{14}$ considered only 33 patients, which is a small sample size for conclusive consideration.

A limit of this review is the lack of data on the influence of knee alignment on cartilage damage in ACL-deficient knees. As reported by Noyes et al, ${ }^{26}$ any combination of conditions leading to higher medial joint forces would be associated with factors leading to more rapid degeneration of the medial compartment in patients with ACL deficiency, varus deformity, and lax lateral ligament structures.

This review has some other limitations. First, time and language restrictions limited literature search to the English literature of the past 10 years. Second, the study solely focused on radiographic aspects of articular degeneration and did not consider the clinical aspects of $\mathrm{OA}$. As reported in the literature, OA after ACL reconstruction causes symptomatic knee problems. Barenius et $\mathrm{al}^{27}$ found symptomatic OA of the medial compartment in $39 \%$ of patients. Lohmander et $\mathrm{al}^{28}$ found $42 \%$ of symptomatic OA in their cohort of female soccer players 12 years after an injury.

In conclusion, the current literature highlights a high risk of osteoarthritic evolution after combined ACL and meniscal tears. Isolated ACL tears treated by arthroscopic reconstruction seem to evolve in cartilage degeneration, but only a little number of high-quality studies focused on these specific patients. Multicenter studies or implementation of national registries focusing on this topic could help to understand the specific influence of ACL tears on osteoarthritic evolution of the knee.

\section{References}

1 Moses B, Orchard J, Orchard J. Systematic review: annual incidence of ACL injury and surgery in various populations. Res Sports Med 2012;20(3-4):157-179

2 Mihata LC, Beutler AI, Boden BP. Comparing the incidence of anterior cruciate ligament injury in collegiate lacrosse, soccer, and basketball players: implications for anterior cruciate ligament mechanism and prevention. Am J Sports Med 2006;34(06):899-904

3 Torg JS, Conrad W, Kalen V. Clinical diagnosis of anterior cruciate ligament instability in the athlete. Am J Sports Med 1976;4(02): 84-93

4 Church S, Keating JF. Reconstruction of the anterior cruciate ligament: timing of surgery and the incidence of meniscal tears and degenerative change. J Bone Joint Surg Br 2005;87(12):1639-1642

5 Goradia VK, Grana WA. A comparison of outcomes at 2 to 6 years after acute and chronic anterior cruciate ligament reconstructions using hamstring tendon grafts. Arthroscopy 2001;17(04):383-392

6 Kennedy J, Jackson MP, O'Kelly P, Moran R. Timing of reconstruction of the anterior cruciate ligament in athletes and the incidence 
of secondary pathology within the knee. J Bone Joint Surg $\mathrm{Br}$ 2010;92(03):362-366

7 Muaidi QI, Nicholson LL, Refshauge KM, Herbert RD, Maher CG. Prognosis of conservatively managed anterior cruciate ligament injury: a systematic review. Sports Med 2007;37(08):703-716

8 van Meer BL, Meuffels DE, van Eijsden WA, Verhaar JA, BiermaZeinstra SM, Reijman M. Which determinants predict tibiofemoral and patellofemoral osteoarthritis after anterior cruciate ligament injury? A systematic review. Br J Sports Med 2015;49(15):975-983

9 Salata MJ, Gibbs AE, Sekiya JK. A systematic review of clinical outcomes in patients undergoing meniscectomy. Am J Sports Med 2010;38(09):1907-1916

10 Cameron M, Buchgraber A, Passler H, et al. The natural history of the anterior cruciate ligament-deficient knee. Changes in synovial fluid cytokine and keratan sulfate concentrations. Am J Sports Med 1997;25(06):751-754

11 Moher D, Liberati A, Tetzlaff J, Altman DG; PRISMA Group. Preferred reporting items for systematic reviews and meta-analyses: the PRISMA statement. J Clin Epidemiol 2009;62(10):1006-1012

12 Kievit AJ, Jonkers FJ, Barentsz JH, Blankevoort L. A cross-sectional study comparing the rates of osteoarthritis, laxity, and quality of life in primary and revision anterior cruciate ligament reconstructions. Arthroscopy 2013;29(05):898-905

13 Jones MH, Spindler KP, Fleming BC, et al. Meniscus treatment and age associated with narrower radiographic joint space width 2-3 years after ACL reconstruction: data from the MOON onsite cohort. Osteoarthritis Cartilage 2015;23(04):581-588

14 Lidén M, Sernert N, Rostgård-Christensen L, Kartus C, Ejerhed L. Osteoarthritic changes after anterior cruciate ligament reconstruction using bone-patellar tendon-bone or hamstring tendon autografts: a retrospective, 7-year radiographic and clinical follow-up study. Arthroscopy 2008;24(08):899-908

15 Hoffelner T, Resch H, Moroder P, et al. No increased occurrence of osteoarthritis after anterior cruciate ligament reconstruction after isolated anterior cruciate ligament injury in athletes. Arthroscopy 2012;28(04):517-525

16 Struewer J, Frangen TM, Ishaque B, et al. Knee function and prevalence of osteoarthritis after isolated anterior cruciate ligament reconstruction using bone-patellar tendon-bone graft: long-term follow-up. Int Orthop 2012;36(01):171-177

17 Kessler MA, Behrend H, Henz S, Stutz G, Rukavina A, Kuster MS. Function, osteoarthritis and activity after ACL-rupture: 11 years follow-up results of conservative versus reconstructive treat- ment. Knee Surg Sports Traumatol Arthrosc 2008;16(05): 442-448

18 Aït Si Selmi T, Fithian D, Neyret P. The evolution of osteoarthritis in 103 patients with ACL reconstruction at 17 years follow-up. Knee 2006;13(05):353-358

19 Leiter JR, Gourlay R, McRae S, de Korompay N, MacDonald PB. Long-term follow-up of ACL reconstruction with hamstring autograft. Knee Surg Sports Traumatol Arthrosc 2014;22(05): 1061-1069

20 Zaid M, Lansdown D, Su F, et al. Abnormal tibial position is correlated to early degenerative changes one year following ACL reconstruction. J Orthop Res 2015;33(07):1079-1086

21 Nakata K, Shino K, Horibe S, et al. Arthroscopic anterior cruciate ligament reconstruction using fresh-frozen bone plug-free allogeneic tendons: 10-year follow-up. Arthroscopy 2008;24(03): 285-291

22 Li RT, Lorenz S, Xu Y, Harner CD, Fu FH, Irrgang JJ. Predictors of radiographic knee osteoarthritis after anterior cruciate ligament reconstruction. Am J Sports Med 2011;39(12):2595-2603

23 Gerhard P, Bolt R, Dück K, Mayer R, Friederich NF, Hirschmann MT. Long-term results of arthroscopically assisted anatomical singlebundle anterior cruciate ligament reconstruction using patellar tendon autograft: are there any predictors for the development of osteoarthritis? Knee Surg Sports Traumatol Arthrosc 2013; 21(04):957-964

24 Deledda D, Rosso F, Cottino U, Bonasia DE, Rossi R. Results of meniscectomy and meniscal repair in anterior cruciate ligament reconstruction. Joints 2016;3(03):151-157

25 McDermott I. Meniscal tears, repairs and replacement: their relevance to osteoarthritis of the knee. Br J Sports Med 2011; 45(04):292-297

26 Noyes FR, Schipplein OD, Andriacchi TP, Saddemi SR, Weise M. The anterior cruciate ligament-deficient knee with varus alignment. An analysis of gait adaptations and dynamic joint loadings. Am J Sports Med 1992;20(06):707-716

27 Barenius B, Ponzer S, Shalabi A, Bujak R, Norlén L, Eriksson K. Increased risk of osteoarthritis after anterior cruciate ligament reconstruction: a 14-year follow-up study of a randomized controlled trial. Am J Sports Med 2014;42(05):1049-1057

28 Lohmander LS, Ostenberg A, Englund M, Roos H. High prevalence of knee osteoarthritis, pain, and functional limitations in female soccer players twelve years after anterior cruciate ligament injury. Arthritis Rheum 2004;50(10):3145-3152 